\title{
Determination of the Xanthate Group Distribution on Viscose by Liquid-State ${ }^{1} \mathrm{H}$ NMR Spectroscopy
}

Andreas Schwaighofer ${ }^{1,2}$, Gerhard Zuckerstätter ${ }^{1}$, Judith Schlagnitweit ${ }^{2}$, Herbert Sixta ${ }^{3}$ and Norbert Müller ${ }^{2, *}$

${ }^{1}$ Kompetenzzentrum Holz GmbH, St.-Peter-Straße 25, 4021 Linz, Austria

${ }^{2}$ Institute of Organic Chemistry, Johannes Kepler University, Altenbergerstraße 69, 4040 Linz, Austria

${ }^{3}$ Department of Forest Products Technology, Helsinki University of Technology, Vuorimiehentie 1, 02015 Espoo, Finland

* author to whom correspondence should be addressed at: norbert.mueller@jku.at, phone: +43732 2468 8746, fax: +43732 24688747

This is a post-peer-review, pre-copyedit version of an article published in Analytical Bioanalytical Chemistry. The final authenticated version is available online at DOI: 10.1007/s00216-010-4570-2.

\section{Abstract}

An analytical method for determination of the xanthate group distribution on viscoses based on liquid-state NMR spectroscopy was developed. Sample preparation involves stabilization of the xanthate group by allylation followed by derivatization of the remaining free hydroxyl groups at the glucose unit.

The method was applied for studying (1) the $\gamma$-value (number of xanthate groups per 100 glucose units) of viscose, (2) the distribution of the xanthate groups on the anhydroglucose unit (AGU), and (3) changes of the xanthate group distribution during ripening. Results of the $\gamma$-value determination are well comparable with reference methods. Elucidation of the xanthate group distribution on the AGU gives the percentage at the C-6 position and a cumulative share of the positions C-2 and C-3. During ripening, xanthate groups at C-2 and C-3 degrade first, while xanthates at C-6 decompose at a slower rate.

Keywords ${ }^{1} H$ NMR, viscose, xanthate group distribution, gamma value 


\section{Introduction}

Although the viscose process [1] was invented in 1891 and accordingly has been known for almost 120 years by now, some aspects are still not fully understood and are a focus of current research. Especially the chemical reactions during the ripening stage are a point of interest as they directly influence subsequent procedure steps as well as the quality of the fibre product. The well-known fact that with decreasing degree of substitution by xanthate groups ( $\gamma$-value) the concentration of by-products increases, does not give a full description of the ripening processes.

A further important parameter is the xanthate group distribution on the cellulose. While the distribution along the cellulose chain and between cellulose molecules has been investigated elsewhere $[2,3,4]$, the motivation of this work is the elucidation of the xanthate group distribution among the C-2, C-3 and C-6 positions of the AGU (anhydroglucose unit). This type of distribution evolves from the dissimilar thermodynamic stability and kinetic reactivity of the xanthate group on the different positions on the AGU. As the $\mathrm{OH}-6$ group possesses the highest mobility and accessibility, it is sterically favored and therefore reacts first in substitution reactions resulting in a high degree of xanthate substitution at $\mathrm{OH}-6$ from the very beginning of xanthation. Also at a primary alcohol group xanthation is both, thermodynamically and kinetically favored. Xanthation at the secondary alcohol groups, $\mathrm{OH}-$ 2 and $\mathrm{OH}-3$, is kinetically and thermodynamically disfavored both, for electronic reasons and due to the sterical crowding in the cellulose chain. Hence $\mathrm{OH}-2$ and $\mathrm{OH}-3$ are hydrolyzed faster during ripening, leading to a decay of the xanthate substitution at $\mathrm{OH}-2\left(\gamma_{2}\right)$ and $\mathrm{OH}-3\left(\gamma_{3}\right)$ and a relative rise of xanthate substitution at OH-6 $\left(\gamma_{6}\right)$ during ripening. A second process that promotes high $\gamma_{6^{-}}$ substitution is trans-xanthation, which preferably transfers xanthate groups from $\mathrm{OH}-2$ to OH-6 [5].

First attempts to examine this distribution on the AGU were made by Lieser [6] already in 1926. Further research was done by Lauer [7], Purves et al. [8], Willard and Pascu [9], as well as Philipp [10], but their results were contradictory or disproved by later publications, e.g.: [11].

More recent analytical approaches are based on NMR spectroscopy. As carbohydrates have a high abundance of hydrogen and carbon, ${ }^{1} \mathrm{H}$ and ${ }^{13} \mathrm{C}$ NMR is a preferred tool for structure elucidation of cellulose and its derivates. The prominent advantage of NMR in quantitative 
analysis derives from the fact, that no compound or group-specific calibration is required, since the relative signal amplitudes (integrals) in a spectrum always directly correlate to relative numbers of nuclei, provided sufficient time for relaxation is given. The latter condition is often posing severe practical restrictions for ${ }^{13} \mathrm{C} N \mathrm{NM}$ but is easily fulfilled for ${ }^{1} \mathrm{H}$ NMR [12].

In 1987, Kamide et al. [11] examined the xanthate distribution by signal analysis of the Osubstituted and O-unsubstituted glucopyranose ring carbons by ${ }^{13} \mathrm{C}$ NMR and provided data for the changes during the ripening process. O-substituted and $\mathrm{O}$-unsubstituted ring carbons show distinctly different chemical shifts, so $\gamma_{2}, \gamma_{3}$ and $\gamma_{6}$-values can be directly calculated from ratios of the respective peak areas, which are, however, small numbers and thus errorprone at the usually low signal-to-noise ratio of ${ }^{13} \mathrm{C}$ NMR at natural isotope abundance (1.1\%). In 1993, König et al. [13] derivatized cellulose xanthate with ${ }^{13} \mathrm{C}$-labelled carbon disulfide, thus bypassing the downside of ${ }^{13} \mathrm{C}$ NMR, which is the low sensitivity due to its low natural abundance. With regard to changes of the substituent distribution during ripening, these results are in accordance with Kamide et al. [11]. But both, Kamide and König worked with self-prepared viscose-samples. Consequently their methods are not directly applicable to industrial viscose. Rußler et al. [14] used GC-analysis of stabilized and methylated industrial viscose samples.

The goal of the present work is to develop a routinely applicable analytical method for determination of the xanthate group distribution on the AGU of industrial viscose samples. The method is based on chemical stabilization of the xanthate group followed by derivatization of the remaining hydroxyl groups. In a comparative derivatization study comprising acetylation, propionylation and trifluoroacetylation, the most satisfying results with respect to solubility, reaction conditions and spectroscopic differentiation, were achieved with acetylation. Acetylated viscose derivatives can be analyzed conveniently by ${ }^{1} \mathrm{H}$ NMR, thereby avoiding the disadvantages and inconvenience of quantitative ${ }^{13} \mathrm{C} N M R$, i.e. low sensitivity despite long accumulation times paired with long $T_{1}$ relaxation times, requiring large inter-acquisition delays.

The first step of derivatization is the stabilization of the xanthate group by allylation [15], primarily to prevent any reactions involving the xanthate groups, thus freezing the xanthate distribution prior to further derivatization. The second purpose of stabilization is the introduction of an easily quantifiable group for the determination of the $\gamma$-value. The subsequent acetylation of the remaining hydroxyl groups also serves a dual purpose. First the 
solubility of the otherwise insoluble (in usual NMR-solvents) allylated viscose is increased thus enabling analysis by solution methods. Second the NMR chemical shifts of the acetyl methyl groups introduced are regio-sensitive with respect to their positions on the AGU rings. The distribution of the xanthate groups on the AGU can then be derived from solution state ${ }^{1} \mathrm{H}$ NMR spectra indirectly via the positions and relative amounts of different acetyl methyl signals.

\section{Materials and Methods}

\section{Solvents and chemicals}

Allyl bromide (reagent grade, 97\%, Sigma-Aldrich), deuterated chloroform (99.8\% D, Sigma-Aldrich), Amberlite IRA 402 ion exchange resin (Sigma-Aldrich), acetic anhydride (p.a., Merck), acetic acid (p.a., Merck), acetone (p.a., Merck), ethanol ( $\geq 99.9 \%$, Merck), diethylether (HPLC grade, FSA Laboratory Supplies), and pyridine (p.a., $\geq 99 \%$, Fluka) were used as purchased.

\section{Sample preparation}

All viscose samples were provided by Lenzing AG. Standard viscose samples used for stabilization and derivatization as well as for the ripening experiment were synthesized by Lenzing R\&D based on a procedure by Treiber [16].

In order to slow down the ripening reactions during stabilization, all of the following steps were carried out in an ice bath. To obtain ca. $300 \mathrm{mg}$ of stabilized viscose, $3.5 \mathrm{~g}$ cellulose xanthate were dissolved in $20 \mathrm{~mL}$ of water under stirring. After addition of $40 \mathrm{~mL}$ Amberlite IRA 402 ion exchange resin, the mixture was stirred vigorously for $15 \mathrm{~min}$. Then the ion exchange resin was separated by suction filtration and washed with de-ionized water $(250 \mathrm{~mL})$. The $\mathrm{pH}$ was set to $7-7.5$ with $20 \%$ acetic acid. To start allylation, $350 \mu \mathrm{L}$ (4.05 mmol) allylbromide were added to the solution. After vigorous stirring in the ice bath for 2.5 hours, the solution was kept at $4{ }^{\circ} \mathrm{C}$ for $15 \mathrm{~h}$. The precipitate (product 1) was separated by suction filtration. The stabilized viscose was washed subsequently with water $(100 \mathrm{~mL})$, water:acetone 1:1 $(40 \mathrm{~mL})$, acetone $(20 \mathrm{~mL})$, and diethyl ether $(15 \mathrm{~mL})$ before drying under vacuum $\left(10^{-2}\right.$ mbar) for 4 hours.

For acetylation of the hydroxyl groups, $200 \mathrm{mg}$ manually grinded product $\mathbf{1}$ was dissolved in $6 \mathrm{~mL}$ pyridine. After adding $6 \mathrm{~mL}(63.5 \mathrm{mmol})$ acetic anhydride, the reaction mixture was 
kept at $60{ }^{\circ} \mathrm{C}$ for $3 \mathrm{~h}$ in $\mathrm{N}_{2}$ atmosphere under stirring. The polymer was precipitated in $100 \mathrm{~mL}$ ethanol:water $1: 1$ and dried under vacuum.

\section{NMR spectroscopy}

For solution NMR measurements, the solid samples were manually grinded and dissolved in $\mathrm{CDCl}_{3}$. Spectra of allylated and acetylated viscoses were recorded with a sample concentration of $0.1 \% \mathrm{w} / \mathrm{w}$.

For signal assignments, $\left[{ }^{1} \mathrm{H},{ }^{13} \mathrm{C}\right]-\mathrm{HSQC}$ and $\left[{ }^{1} \mathrm{H},{ }^{13} \mathrm{C}\right]-\mathrm{HMBC}$ spectra of stabilized and acetylated viscose were recorded on a $500 \mathrm{MHz}$ Bruker DRX NMR spectrometer equipped with a cryogenically cooled triple resonance probe (TXI). The HSQC NMR spectrum was recorded with $4 \mathrm{~K}\left({ }^{1} \mathrm{H}\right.$, direct dimension, $16 \mathrm{ppm}$ spectral width) times $256\left({ }^{13} \mathrm{C}\right.$, indirect dimension, 220 ppm spectral width) real data points, 80 accumulations per increment, $1 \mathrm{~s}$ relaxation delay and $0.26 \mathrm{~s}$ acquisition time. The HMBC NMR spectrum comprised $4 \mathrm{~K}\left({ }^{1} \mathrm{H}\right.$, direct dimension, $16 \mathrm{ppm}$ spectral width) times $256\left({ }^{13} \mathrm{C}\right.$, indirect dimension, $220 \mathrm{ppm}$ spectral width) real data points, 600 accumulations per increment, $1 \mathrm{~s}$ relaxation delay and $0.26 \mathrm{~s}$ acquisition time.

Solid-state NMR measurements of stabilized viscose were recorded on a $300 \mathrm{MHz}$ Bruker Avance DPX spectrometer with a $7 \mathrm{~mm}{ }^{1} \mathrm{H} / \mathrm{BB}$ MAS probe at a sample spinning speed of $4 \mathrm{kHz}$ and typically involved the following acquisition parameters: acquisition time: $51 \mathrm{~ms}$, cross polarization (CP) contact time: $1 \mathrm{~ms}, 16384$ accumulations, relaxation delay $\mathrm{d} 1=3 \mathrm{~s}$, $90^{\circ}{ }^{1} \mathrm{H}$ pulse: $5.0 \mu \mathrm{s}, 50 \mathrm{kHz}$

${ }^{1} \mathrm{H}$ decoupling rf-field, line broadening: $20 \mathrm{~Hz}$.

For quantification purposes, all stabilized and acetylated viscose NMR spectra were recorded in $\mathrm{CDCl}_{3}$ on a $300 \mathrm{MHz}$ Bruker Avance DPX spectrometer using a $5 \mathrm{~mm}$ BBI inverse probe equipped with a z-gradient at a temperature of $300 \mathrm{~K}$. Chemical shifts were referenced to the residual solvent signal at $\delta\left({ }^{1} \mathrm{H}\right)=7.26 \mathrm{ppm}$. ${ }^{1} \mathrm{H} T_{1}$ relaxation times were checked with the inversion recovery technique and ranged from 2.0 to $2.2 \mathrm{~s}$ for allyl olefinic protons and 0.8 to $1.1 \mathrm{~s}$ for carbohydrate $\mathrm{CH}$ and $\mathrm{CH}_{2}$, aliphatic allyl- $\mathrm{CH}_{2}$ and acetyl- $\mathrm{CH}_{3}$ protons. To ensure quantitative measurement conditions, one-dimensional ${ }^{1} \mathrm{H}$ NMR spectra were recorded with a $30^{\circ}$ flip-angle ( $90^{\circ}$ pulse: $\left.7.9 \mu \mathrm{s}\right), 4.2 \mathrm{~s}$ acquisition time, $10 \mathrm{~s}$ relaxation delay, and $26 \mathrm{ppm}$ spectral width. For each quantitative ${ }^{1} \mathrm{H}$ NMR spectrum 128 or 256 transients were accumulated. Processing of quantitative ${ }^{1} \mathrm{H}$ NMR spectra involved exponential window multiplication $(\mathrm{LB}=0.3 \mathrm{~Hz}$, to avoid truncation artefacts from slowly relaxing solvent and 
impurity signals without introducing any measurable broadening in the methyl range) of the free induction decay (FID) prior to Fourier transform. Zero-order phase correction and automatic $5^{\text {th }}$ order polynomial baseline correction yielded the final spectrum. All 1D and 2D NMR data were processed and analyzed using the Bruker software TopSpin 2.1.

\section{Results and Discussion}

\section{Allylation and Acetylation}

After xanthation, positions and numbers of the xanthate groups at the AGU change at elevated temperatures and extreme $\mathrm{pH}$ values [15]. Therefore stabilization has to be provided in order to allow reproducible analysis of a sample's original xanthation state. Among known reagents proposed for that purpose $[8,9,15]$, allyl bromide was chosen, because of its favorable reaction characteristics and superior interpretability of NMR spectra, owed to its small molecule size. The polymeric derivatization product is an insoluble white powder, which can be stored at room temperature without further change of composition.
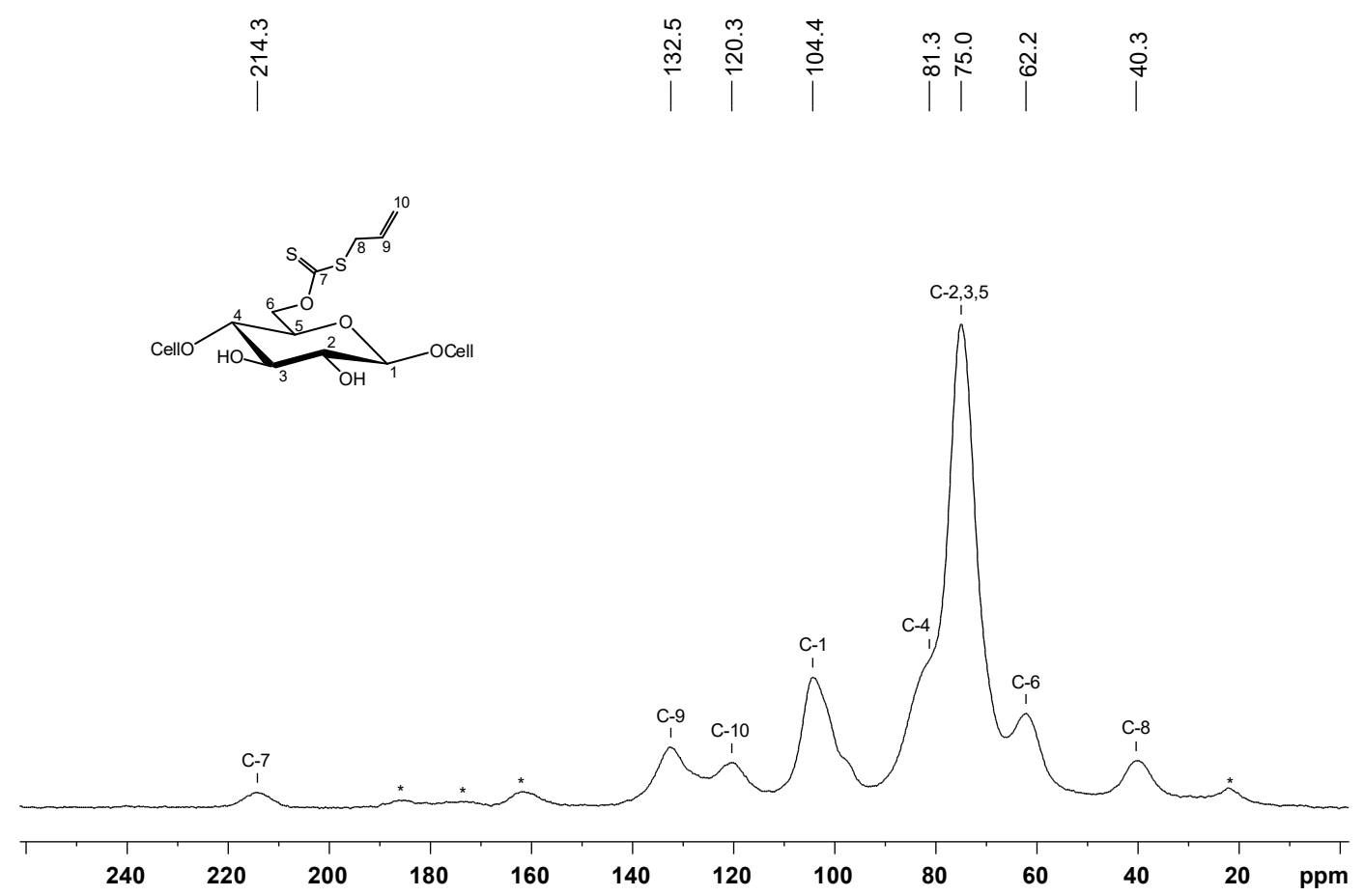

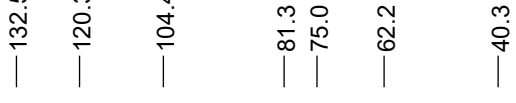

Fig. $1{ }^{13} \mathrm{C}$ NMR CP-MAS spectrum of allylated cellulose xanthate. Spinning side bands are marked by "*" 
There are two important prerequisites for a routinely applicable stabilization: 1. Virtually all xanthate groups on the AGU need to be allylated. 2. No xanthate groups should be cleaved off the AGU during allylation i.e. the $\gamma$-value (i.e. number of xanthate groups per 100 glucose units) must not change while stabilization. The first point can directly be proved by solid state NMR. The ${ }^{13} \mathrm{C}$ NMR shifts of the non-allylated xanthate group carbons appear in the region of 232-234 ppm [13]. The absence of signals in this region of the ${ }^{13} \mathrm{C}$ NMR CP-MAS spectra (see Figure 1) of our stabilized viscose leads to the conclusion that all xanthate groups are allylated.

The second point cannot be directly proved, since neither native nor stabilized viscoses are directly accessible to liquid state NMR investigations. In fact, the $\gamma$-value cannot be determined before derivatization of the remaining free hydroxyl groups. But it can be shown that the $\gamma$-value remains constant throughout the stabilization and derivatization processes by comparison with IR-spectroscopy as a reference method.

For determination of the xanthate group distribution, we derivatized the free hydroxyl groups. This approach has also been successfully applied for determination of the substituent distribution on starch [17] and cellulose derivates [18-21].

After derivatization the hydroxyl groups can be indirectly quantified by the NMR-response of the derivating groups introduced. This implies an increase in sensitivity by approximately a factor of five, since at a typical $\gamma$-value of 50, there are five times more free hydroxyl than xanthate groups on the AGU. After quantification of the hydroxyl groups, the amount and positions of xanthate groups can be calculated as shown below. Propionylation and trifluoroacetylation were also tested as alternative strategies. But they gave results clearly inferior to acetylation with respect to both, reaction conditions and spectrum quality. In the course of optimization, an ideal reaction time of $3 \mathrm{~h}$ at a temperature of $60{ }^{\circ} \mathrm{C}$ was found.

\section{${ }^{1} \mathrm{H}$ NMR spectra and peak assignment}

Figure 2 shows a representative ${ }^{1} \mathrm{H}$ NMR spectrum of allylated and acetylated viscose. Peak assignment was achieved with $\left[{ }^{1} \mathrm{H},{ }^{13} \mathrm{C}\right]$-HSQC and $\left[{ }^{1} \mathrm{H},{ }^{13} \mathrm{C}\right]-\mathrm{HMBC}$ spectra shown in Figure 3 and agrees with literature $[22,23]$. The peaks were assigned as follows: $\delta(\mathrm{ppm})=1.89$, 1.94 and $2.00\left(\mathrm{H}-11_{2,3}\right.$, acetyl- $\left.\mathrm{CH}_{3}\right), 2.12\left(\mathrm{H}-11_{6}\right.$, acetyl- $\left.\mathrm{CH}_{3}\right), 3.52\left(\mathrm{H}_{5}\right.$ on $\left.\mathrm{AGU}\right), 3.71\left(\mathrm{H}_{4}\right.$ on AGU), 3.85 (H-7, allyl H), 4.05 ( $\mathrm{H}_{6}$ ' on AGU), 4.32 ( $\mathrm{H}_{6}$ on AGU), 4.40 ( $\mathrm{H}_{1}$ on AGU), $4.79\left(\mathrm{H}_{2}\right.$ on $\left.\mathrm{AGU}\right), 5.06\left(\mathrm{H}_{3}\right.$ on $\left.\mathrm{AGU}\right), 5.20$ (H-9, allyl H), 5.33 (H-10, allyl H), 5.85 (H-8, allyl $\mathrm{H}_{2}$ ). 
Initially, it was expected that the signal in the region of the acetyl resonances would contain three main peaks corresponding to the acetyl groups in the positions 2, 3 and 6 of the AGU. Surprisingly the acetyl resonances give four resolved peaks with apparent shoulders, indicating a more complex dependence on the AGU substitution pattern. A similar pattern was identified previously during investigations on cellulose acetates (CA) [21]. While the acetyl region of cellulose triacetate (CTA) shows three well defined peaks, each corresponding to one position on the AGU, the splitting pattern in the acetyl region of partially acetylated cellulose seems more complicated with additional peaks and shoulders. It was found that the irregular structure of partially acetylated CA is responsible for this more complicated spectrum. As in CTA every single AGU is tri-acetylated, the same structural unit is repeated along the cellulose chain constituting three chemically different acetyl groups which represent the positions 2, 3 and 6 on the AGU. In partially acetylated CA, three different mono-acetylated and three different di-acetylated AGUs are possible in addition to the tri-acetylated AGU, giving seven different AGU substitution patterns total. The seven different cellulose monomer units can implicate up to twelve distinct acetyl methyl proton resonances [24]. These considerations can be transferred to the spectrum of acetylated stabilized viscose as in that compound acetylation cannot be complete due to the presence of the xanthate group.

Using the HSQC and HMBC NMR spectra shown in Figure 3, the four acetyl peaks in acetylated stabilized viscose were assigned to their positions on the AGU. The peak at 2.12 ppm solely correlates with the C-6 carbon, so it can be assigned to the acetyl group located on C-6 of the AGU. The peaks at 2.00 ppm, 1.94 ppm and 1.89 ppm correlate with the $\mathrm{C}-2$ carbon as well as with the $\mathrm{C}-3$ carbon of the AGU. With regard to the intended quantification, this ambiguous assignment was acceptable and the three peaks are jointly assigned to the acetyl groups on $\mathrm{C}-2$ and $\mathrm{C}-3$ of the AGU to form a cumulative parameter. The clear discrimination of the $\mathrm{H}-11_{6}$ signal from the $\mathrm{H}-11_{2,3}$ signals in the ${ }^{1} \mathrm{H}$ NMR spectrum can be well rationalized by their different chemical environments, as the acetyl group at position 6 is attached to a secondary alcohol group and separated by a methylene group from the AGU ring. 


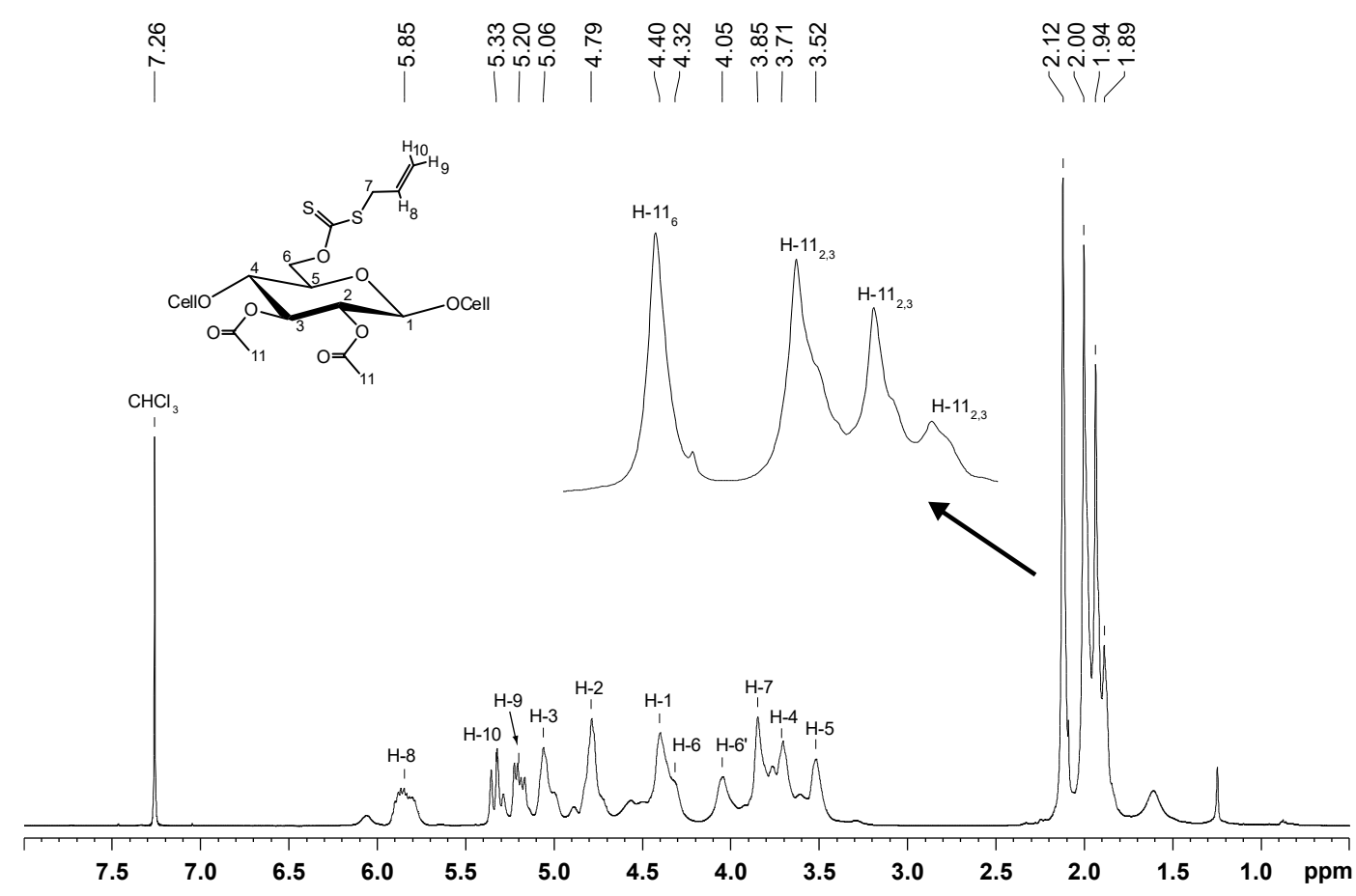

Fig. $2{ }^{1} \mathrm{H}$ NMR spectrum of allylated and acetylated viscose with a close-up of the acetyl region between 1.85$2.25 \mathrm{ppm}$. For reference, the inset structure diagram depicts an AGU, which is xanthated and allylated in position 6 and acetylated in positions 2 and 3

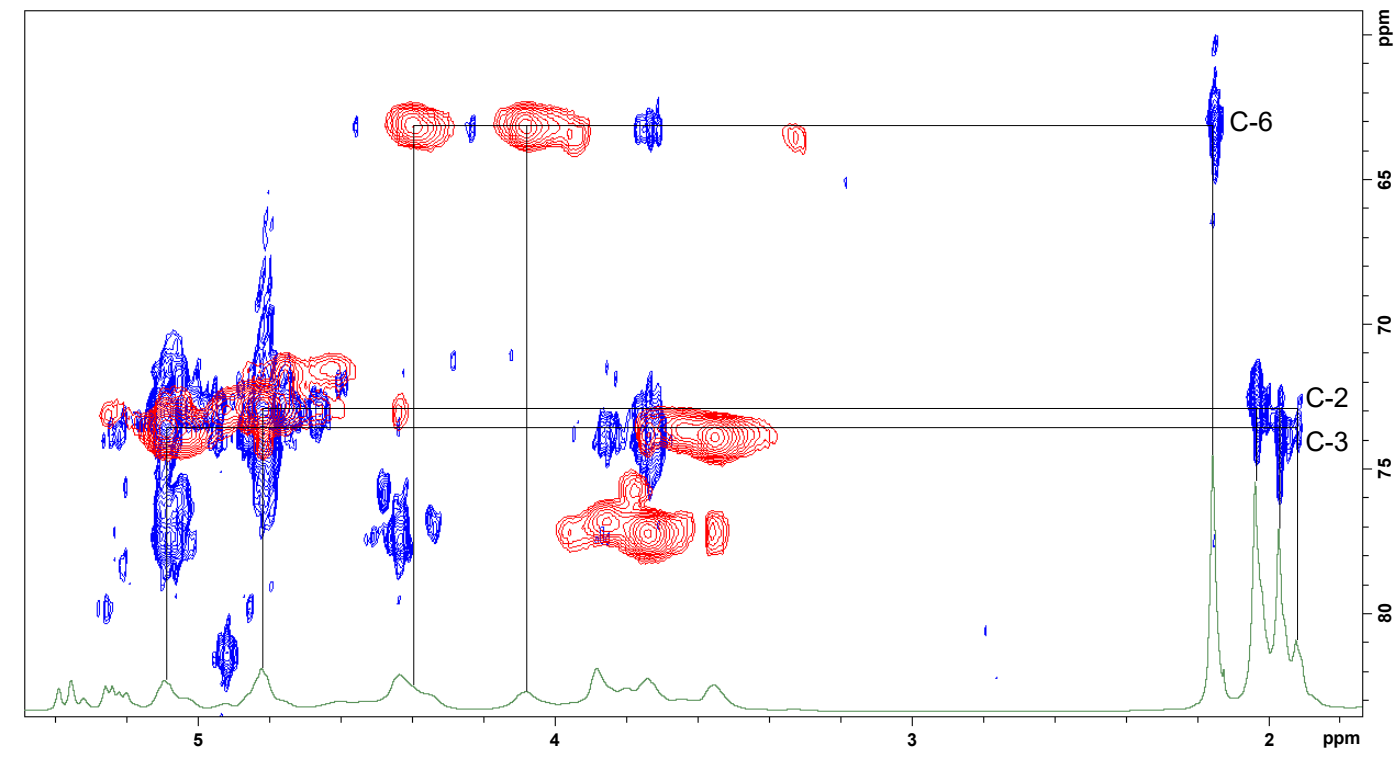

Fig. 3 Overlay of a $\left[{ }^{1} \mathrm{H},{ }^{13} \mathrm{C}\right]$-HSQC spectrum (blue), a $\left[{ }^{1} \mathrm{H},{ }^{13} \mathrm{C}\right]-\mathrm{HMBC}$ spectrum (red) and a ${ }^{1} \mathrm{H}$ NMR spectrum (green) of allylated and acetylated viscose. The thin black lines indicate coupling connectivities 


\section{Determination of the $\gamma$-value}

The degree of xanthation is commonly described by the $\gamma$-value which denotes the number of xanthate groups per 100 anhydroglucose units. During the viscose process, it is routinely determined by iodometric wet chemical analysis [25] respectively UV- and IR-spectroscopic techniques [16, 26, 27]. These methods require careful calibration. Therefore a calibration free method is of substantial advantage. NMR analyses with prior derivatization can be considered as primary methods per definition, since the peak area directly corresponds to the number of nuclei contributing to the signal. Thus there are no response factors involved in quantification. Consequently there is no need for calibration, when evaluating relative peak proportions. Potentially interfering viscose by-products are separated by ion exchange during stabilization and impurities are minimized by washing steps after allylation and acetylation. Since the xanthate groups are not directly accessible for ${ }^{1} \mathrm{H}$ NMR analysis, they are quantified by way of the attached allyl groups in the NMR spectrum as given by equation (1).

$$
\gamma=100 \frac{A_{\text {Xanthate }}}{A_{A G U}}=100 \frac{A_{H-9}+A_{H-10}}{A_{H-2}+A_{H-3}}
$$

Here the subscripts refer to the atom numbers in Fig. 2. H-9 and H-10 of the allyl group represent the xanthate group. The anhydroglucose unit is represented by the integrals of $\mathrm{H}-2$ and $\mathrm{H}-3$ in the calculation.

A comparison of repeated $\gamma$-value analyses by NMR and reference methods is shown in Table 1. For critical evaluation of the NMR method, repeated analyses of a viscose sample were carried out and compared with reference methods. The results demonstrate the good reproducibility of the NMR method through rather low values of the standard deviation, which are below what has been found for the IR method. The standard prediction error of the IR method has been indicated as 1.39 [27]. It should be noted that $\gamma$-values from the NMR measurements are repeatedly and reproducibly higher than with iodometric analysis, while the IR technique gives the lowest $\gamma$-values. 
Table 1 Results of repeated $\gamma$-value determinations of viscose. For NMR analyses, allylation and acetylation steps were carried out separately for every single sample

\begin{tabular}{|c|c|c|c|c|c|c|c|c|c|}
\hline $\begin{array}{c}\text { analysis } \\
\text { method }\end{array}$ & \multicolumn{7}{|c|}{$\gamma$-value } & average & $\begin{array}{l}\text { standard } \\
\text { deviation }\end{array}$ \\
\hline & & & & & & & & & \\
\hline NMR & 55.3 & 55.4 & 55.7 & 54.9 & 55.8 & 55.7 & 54.6 & 55.3 & 0.45 \\
\hline IR & 53.0 & 53.5 & 54.7 & & & & & 53.7 & 0.87 \\
\hline
\end{tabular}

\section{Determination of the substituent distribution}

By quantification of the acetyl groups in the ${ }^{1} \mathrm{H}$ NMR spectrum, an inverse pattern of the xanthate distribution is obtained. Since the peaks of the acetyl region could not be unambiguously assigned to the positions 2, 3 and 6 of the AGU, the acetyl peaks from the positions 2 and 3 are jointly quantified to form a cumulative parameter. Even if a full description of the xanthate distribution is not achievable yet, it is possible to distinguish xanthates on the primary alcohol group (C-6) and xanthates on the secondary alcohol groups (C-2 and C-3). This allows kinetic investigations on reactions occurring during viscose formation and changes during ripening.

In order to obtain reproducible and accurate peak areas, deconvolution analysis of the signals in the acetyl region was carried out as shown in Figure 4. Deconvolution was done with OriginLab's Origin 8 Software [28] using absorptive Lorentzian peak shapes. Seven peak positions were predefined, one for the $\mathrm{CH}_{3}-6$ signal and two (one for the main peak and one for the peak shoulder) for each of the three $\mathrm{CH}_{3}-2,3$ signals. Peak widths were optimized by the fitting routine.

With the deconvoluted peak areas, the distribution of the xanthate groups on the AGU was calculated using equations (2) - (7).

$$
\begin{gathered}
D S_{\text {Acetylation, } x}[\%]=100 \frac{A_{x}}{A_{2,3}-A_{6}} \\
F_{\text {Acetylation, } x}[\%]=D S_{\text {Acetylation, } x} \frac{300-\gamma}{300} \\
F_{\text {Xanthation, } 6}[\%]=33.3-F_{\text {Acetylation, } 6} \\
F_{\text {Xanthation }, 2,3}[\%]=66.6-F_{\text {Acetylation, }, 3}
\end{gathered}
$$




$$
\begin{gathered}
D S_{\text {Xanthation }, x}[\%]=100 \frac{F_{\text {Xanthation }, x}}{F_{\text {Xanthation, }, 3}+F_{\text {Xanthation, } 6}} \\
\gamma_{x}=D S_{\text {Xanthation }, x \cdot \gamma}(7)
\end{gathered}
$$

Results of repeated $\gamma$-value determinations of viscose are summarized in Table 2.

Table $2 \gamma$-value, DS Acetylation, $x$ as well as $\gamma_{6}$ and $\gamma_{2,3}$-values for viscose. For NMR analyses, allylation and acetylation steps were carried out separately for every single sample

\begin{tabular}{ccccccc}
\hline \multirow{2}{*}{$\gamma$-value } & \multicolumn{2}{c}{ DS Acetylation, $^{2}[\%]$} & \multicolumn{2}{c}{ DS Xanthation, $[\%]$} & $\gamma_{6}$ & $\gamma_{2,3}$ \\
& C-6 & C-2,3 & C-6 & C-2,3 & & \\
\hline 55.6 & 29.1 & 70.9 & 51.9 & 48.1 & 28.9 & 26.7 \\
56.1 & 28.9 & 71.1 & 52.6 & 47.4 & 29.5 & 26.6 \\
56.2 & 28.7 & 71.3 & 53.4 & 46.6 & 30.0 & 26.2 \\
\hline
\end{tabular}

With equation (2), the relative distribution of the acetyl groups for the respective positions (DS Acetylation) is calculated from the deconvoluted peak areas (A). After that, the fraction of acetylation in total substitution ( $\left.\mathrm{F}_{\text {Acetylation }}\right)$ is calculated with equation (3).

Equations (4) and (5) express the fraction of xanthation in total substitution ( $\left.F_{\text {Xanthation }}\right)$. The maximum fraction of substitution per position is $33.3 \%$ (equation (4)), thus $66.6 \%$ states the maximum fraction of substitution of two positions in equation (5). This is a critical step in the evaluation, since small variations of $\mathrm{DS}_{\text {Acetylation }}$ lead to larger deviations of $\mathrm{DS}_{\text {Xanthation. }}$ Due to the smaller total amount of xanthate groups, minor differences have a bigger effect here. Thus reproducible peak areas are crucial to obtain repeatable and accurate results. With the automated spectrum post processing and deconvolution routines, this requirement can be met. The relative distribution of the xanthate group on the AGU (DSXanthation) is calculated with equation (6). Finally, the $\gamma$-value for the respective positions on the AGU is calculated with equation (7). 
Our results for $\gamma_{2,3}$ and $\gamma_{6}$-values concur with others' work $[11,13]$ in terms of higher xanthation on C-6 of the AGU. The substitution on the sterically better accessible C-6 position is higher than the collective fraction of $\gamma_{2,3}$. This corroborates the assumption that xanthation is governed by preferred accessibility.

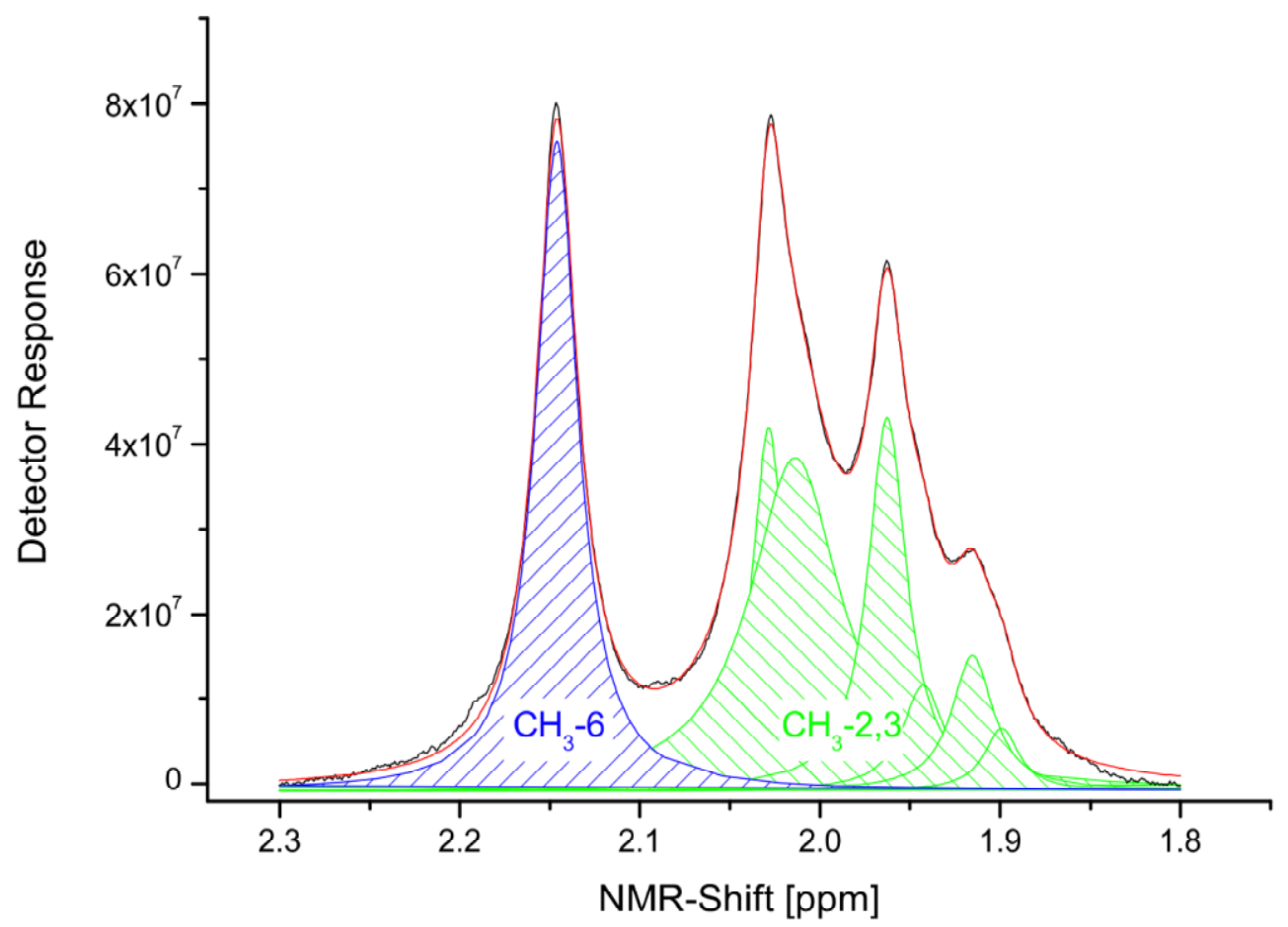

Fig. 4 Deconvolution of peaks in the acetyl region. (black: experimental spectrum; blue, green: fitted signals; red: accumulated fitted signals). A total of seven peaks (one for the $\mathrm{CH}_{3}-6$ signal and two for each of the three $\mathrm{CH}_{3}-2,3$ signals) were used for curve fitting

\section{Changes of the Xanthate Distribution during the Ripening Process}

The analysis method presented above provides a tool for investigating the changes of the substituent distribution on the AGU during ripening, which is important information for the description of the process with its complex reactions. For the experiment, a viscose sample was ripened at $20^{\circ} \mathrm{C}$ in the absence of oxygen and samples were taken for stabilization and derivatization at defined times. Figure 5 shows the course of the $\gamma$-value as well as of the $\gamma_{6}$ and $\gamma_{2,3}$-values with ripening time. The $\gamma$-value generally decreases during ripening due to 
reactions of sodium hydroxide with the xanthate groups, which leads to an increased concentration of inorganic salts, mainly $\mathrm{Na}_{2} \mathrm{CS}_{3}$, in the viscose [16].

The results reveal a rapid drop of xanthate groups on positions 2 and 3 of the AGU.

Hydrolysis of these secondary alcohol groups proceeds up to 20 times faster than on the primary alcohol group on C-6 [10]. The $\gamma_{6}$-value decreases more slowly and even appears to pass through a maximum. A potential explanation for that phenomenon was presented by Trimnell et al. [5], who proposed trans-xanthation reactions within the AGU from positions 2 and 3 to C-6. Also rearrangement of xanthate groups along the cellulose chain would explain this experimental outcome, since the C- 6 position would be the preferred target position due to its higher accessibility.

As a consequence of the fast decrease of $\gamma_{2,3}$ and the relative invariance of the $\gamma_{6}$, xanthate groups on C-2 and C-3 were almost completely hydrolyzed within 70 hours. Hence, the overall $\gamma$-value becomes equivalent to $\gamma_{6}$. Comparable results were found in earlier studies about viscose ripening [13].

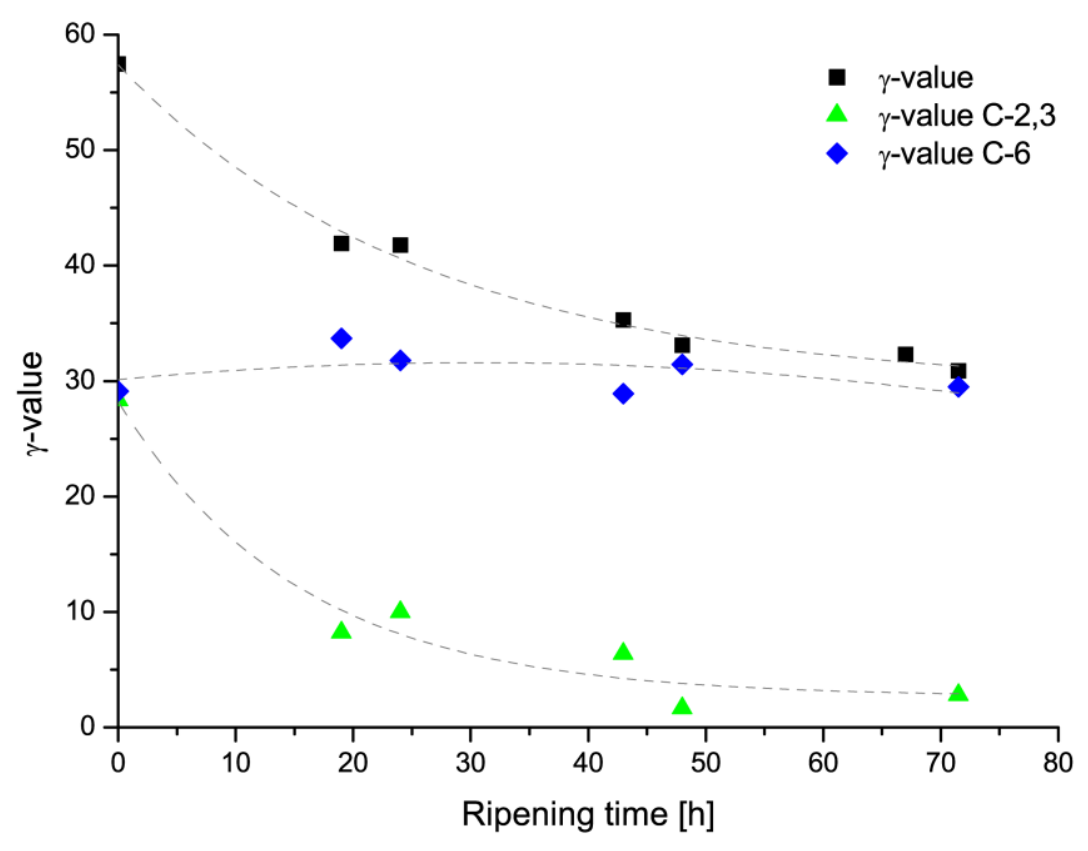

Fig. 5 Development of $\gamma$-value as well as $\gamma_{6}$ and $\gamma_{2,3}$ during ripening. The dashed lines indicate exponential fitting. $\gamma_{2,3}$ decreases faster due to higher affinity to hydrolysis as well as trans-xanthation [5], which even leads to an increase of $\gamma_{6}$ 


\section{Conclusion}

The newly introduced analytical method based on ${ }^{1} \mathrm{H}$ NMR of derivatized cellulose-xanthate allows the determination of the substituent distribution on the anhydroglucose unit of industrial viscose samples. This approach involves acetylation of the free hydroxyl groups on viscose after stabilization of the xanthate group. With only two reaction steps and short ${ }^{1} \mathrm{H}$ NMR acquisition times, this technique is apt for routine application. As a calibration free method it also may serve to optimize other analytical protocols, which are routinely used for this purpose. In further studies, this procedure will also allow investigations of the influence of various process parameters on the xanthate distribution.

\section{Acknowledgments}

Financial support was provided by the Austrian government, the provinces of lower Austria, upper Austria, and Carinthia as well as by Lenzing AG. We also express our gratitude to the University of Natural Resources and Applied Life Sciences, Vienna, and Lenzing AG for their kind contribution. 


\section{References}

[1] Cross CF, Bevan EJ, Beadle C (1893) Dissolution of cotton or wood cellulose as cellulose xanthate. Patent no: GB 8700

[2] Fischer K, Krasselt K, Schmidt I, Weightman D (2005) Distribution of Substituents along the Cellulose Chain on Cellulose Xanthate and Carboxymethyl Cellulose. Macromol Symp 223:109-120

[3] Fischer K, Schmidt I, Hintze H (1994) Untersuchungen zur Substituentenverteilung in Cellulosexanthogenat. Das Papier 12:769-774

[4] Rußler A (2005) Substituentenverteilung an Cellulosexanthogenaten. Dissertation. Universität Hamburg, Wien

[5] Trimnell D, Doane WM, Russell CR, Rist CE (1967) Migration of thiolthiocarbonyl groups of methyl [alpha]-glucopyranoside xanthates. Carbohydr Res 5:166-175

[6] Lieser T (1928) Die Konstitution des Cellulose-Xanthogenats. Liebigs Ann Chem 464:43-55

[7] Lauer K (1950) Zur Kenntnis der Cellulosefasern. 14. Mitteilung: Die Verteilung der Xanthogenatgruppen in heterogenen xanthogenierten Cellulosen. Makromol Chem 5:287-291

[8] Purves CB, Sanyal AK, Falconer EL, Vincent DL (1957) Attempted Location of the Substituent in Cellulose Xanthate by new Methods. Can J Chem 35:1164-1173

[9] Willard JJ, Pacsu E (1960) Location of Xanthate Groups in Viscose. J Am Chem Soc 82:4347-4349

[10] Philipp B, Bär HJ, Dautzenberg H (1966) Kinetische Untersuchungen zur Umxanthogenierung von Cellulosexanthogenat. Faserforsch Textiltechn 17:551-558

[11] Kamide K, Kowsaka K, Okajama K (1987) ${ }^{13}$ C NMR Study on the Distribution of Substituent Groups on Trihydric Alcohol Groups in Cellulose Xanthate. Polym J 19:231-240

[12] Pauli GF, Jaki BU, Lankin DC (2005) Quantitative ${ }^{1} \mathrm{H}$ NMR: Development and Potential of a Method for Natural Products Analysis. J Nat Prod 68:133-149

[13] König L, Döring R, Postel S (1993) ${ }^{13} \mathrm{C}-\mathrm{NMR}$-Untersuchungen an ${ }^{13} \mathrm{C}$-markierten Cellulosexanthogenaten. Das Papier 11:641-644

[14] Rußler A, Lange T, Potthast A, Rosenau T, Berger-Nicoletti E, Sixta H, Kosma P (2005) A Novel Method for Analysis of Xanthate Group Distribution in Viscoses. Macromol Symp 223:189-199

[15] Lange T, Berger-Nicoletti E, Kosma P, Potthast A, Sixta H (2003) Untersuchungen zur Bestimmung der Substituentenverteilung bei Viskosen. Lenzinger Berichte 82:102-106

[16] Götze K (1967) Chemiefasern nach dem Viskoseverfahren. Springer-Verlag, Berlin, Heidelberg, New York

[17] Dicke R, Rahn K, Haack V, Heinze T (2001) Starch derivatives of high degree of functionalization. Part 2. Determination of the functionalization pattern of p-toluenesulfonyl starch by peracylation and NMR spectroscopy. Carbohydr Polym 45:43-51

[18] Iwata T, Azuma J, Okamura K, Muramoto M, Chun B (1992) Preparation and NMR assignments of cellulose mixed esters regioselectively substituted by acetyl and propanoyl groups. Carbohydr Res 224:277-283 [19] Friebolin H, Deus C (1991) Partiell acetylierte Cellulose - Synthese und Bestimmung der Substituentenverteilung mit Hilfe der ${ }^{1}$ H NMR Spektroskopie. Makromol Chem 192:75-83 
[20] Tezuka Y, Imai K, Oshima M, Chiba T (1987) Determination of substituent distribution in cellulose ethers by means of a ${ }^{13} \mathrm{C}$ NMR study on their acetylated derivatives. Macromolecules 20:2413-2418

[21] Goodlett VW, Dougherty JT, Patton HW (1971) Characterization of Cellulose Acetates by Nuclear Magnetic Resonance. J Polym Sci, Part A: Polym Chem 9:155-161

[22] Friebolin H, Keilich G, Seifert E (1969) Protonenresonanz-Untersuchungen an PolysaccharidDerivaten. Angew Chem 81:791-792

[23] SDBSWeb : http://riodb01.ibase.aist.go.jp/sdbs/ (National Institute of Advanced Industrial Science and Technology, 06/13/2010)

[24] Buchanan CM, Edgar KJ, Hyatt JA, Wilson AK (1991) Preparation of Cellulose [1- ${ }^{13}$ C] Acetates and Determination of Monomer Composition by NMR Spectroscopy. Macromolecules 24:3050-3059

[25] Zellcheming Merkblatt III/21/72 (1972) Veresterungsgrad (Gammawert) des Cellulose-xanthogenates nach dem Ionenaustausch-Verfahren (Batch-Methode). Verein Zellcheming, Darmstadt

[26] Klemm D, Philipp B, Heinze T, Heinze U, Wagenknecht W (1998) Comprehensive Cellulose Chemistry. Volume 1 - Fundamentals and Analytical Methods. Wiley-VCH, Weinheim

[27] Baldinger T, Moosbauer H, Jary S (2003) Method for the spectroscopic analysis of viscose constituents. Patent no: WO 03/062818

[28] OriginLab OriginPro 8G SR5, v8.0987 (Build 987) 